\title{
Ergojump evaluation of the explosive strength in volleyball athletes pre- and post-fascial treatment
}

\author{
ANDREA BUSCEMI $^{1}$, MARIA CRISTINA PETRALIA ${ }^{2}$, TIZIANA RAMACI ${ }^{3}$, \\ ALESSANDRO RAPISARDA ${ }^{1}$, CARMELO PROVAZZA ${ }^{1}$, DONATELLA DI CORRADO ${ }^{3}$, \\ VINCENZO PERCIAVALLE ${ }^{4}$, VALENTINA PERCIAVALLE ${ }^{5}$ and MARINELLA COCO ${ }^{4}$ \\ ${ }^{1}$ Department of Research, Center Studies of Osteopathy, I-95129 Catania; ${ }^{2}$ Bonino Pulejo Neurolesi Center, \\ IRCCS, I-98124 Messina; ${ }^{3}$ Sciences of Man and of The Society, University Kore of Enna, I-94100 Enna; \\ Departments of ${ }^{4}$ Biomedical and Biotechnological Sciences and ${ }^{5}$ Educational Sciences, \\ University of Catania, I-95123 Catania, Italy
}

Received October 8, 2017; Accepted December 28, 2018

DOI: $10.3892 / \mathrm{etm} .2019 .7628$

\begin{abstract}
It has previously been demonstrated that physiological mechanisms are involved in muscle pain and fatigue, as the nociceptive afferents of the fascial system are able to modulate the afferent response of the central nervous system. The purpose of the present study was to evaluate a sample of volleyball players, and investigate whether osteopathic treatment of the lower limb muscle groups improved the explosive force of the limbs, whilst reducing spasms and tension, releasing tissue strain and correcting posture. A randomized control study was performed to evaluate 57 athletes who underwent fascial manipulative treatment to assess if such treatment affected the muscle strength of the lower limbs. The treatment group demonstrated a statistically significant improvement in the squatting jump test $(\mathrm{P}<0.0001)$ and in the counter movement jump test $(\mathrm{P}<0.0001)$. Furthermore, the control group did not exhibit any improvement in the squatting jump test $(\mathrm{P}<0.56)$ or in the counter movement jump test $(\mathrm{P}<0.32)$. The results suggested that correction of the fascial system required a minimum time of 30 days in order to obtain an improvement of fascial mechanics and sports performance. Therefore, use
\end{abstract}

Correspondence to: Dr Andrea Buscemi, Department of Research, Center Studies of Osteopathy, Via Vecchia Ognina 141H, I-95129 Catania, Italy

E-mail: andreabuscemi@virgilio.it

Dr Marinella Coco, Department of Biomedical and Biotechnological Sciences, University of Catania, Via Santa Sofia 89, I-95123 Catania, Italy

E-mail: marinella.coco@unict.it

Abbreviations: FTG, fascial treatment group; CG, control group; PFT, protocol of osteopathic fascial techniques; FT, flight time; SJ, squatting jump; CMJ, counter movement jump

Key words: Ergojump, fascial treatment, explosive strength, volleyball athletes of a fascial protocol during athletic training will help improve the balance of the bands and, as a direct consequence, improve the efficiency of the musculoskeletal system, thereby reducing the risk of injury. It would therefore be advisable to perform osteopathic treatment techniques every two months during an athletic season to maintain the balance of the fascial system and obtain the most efficient results.

\section{Introduction}

Force is an innate ability of a body that enables an individual to modify their body and environment. It is possible to produce muscle power through effectors muscles. The proper function of muscles allows for them to efficiently express adequate levels of strength and forms the basis of health and well-being in individuals. Furthermore, muscle strength is indispensable for proper performance in sports and inevitably affects the practice of any sports disciplines. Numerous studies (1-7) have identified a significant role of the connective tissue that surrounds muscles during contraction. Branislav et al (8), hypothesized that the force produced by muscle causes a deformation of the surrounding connective tissue that affects strength. The transmission of muscle strength is made possible by the contiguous nature of the fascia as well as its integrity. These features facilitate the transmission of force, which results in motor activity (7,9-11). Bordoni and Zanier (2) stated that 'connective tissue can control the orientation of the muscle fibres, so as to reflect the vector of the direction of the force, and to make the transition of the more fluid and ergonomic voltage'.

Fascial tissue alterations may induce various symptoms that influence the quality of life of the subject (12-13). In many instances, these symptoms are debilitating and difficult to detect using conventional diagnostic tests (14-17). For example, chronic fatigue is a common symptom (18). Recent studies have demonstrated that many physiological mechanisms are involved in the development of muscle pain and fatigue, as the nociceptive afferents of the fascial system are able to modulate the afferent response of the central nervous system (18).

In the present study, we examined athletes who play volleyball, a sport for which strength is needed and jumping 
is needed too, this could be mentioned here as strength is needed in many sports, but jumping is specific to volleyball. Many authors have examined the concept of training of explosive strength (19-23) and have concluded that jumping ability can be a decisive factor in succeeding in volleyball as it facilitates both attack and in defense (24-27). Studies by Newton et al $(28,29)$ demonstrated that performing specific training tasks increases power and strength of the muscles of the lower limbs, thereby resulting in improvements in the height achieved during a vertical jump.

The main goal of the present study was to evaluate if undergoing specific osteopathic treatment of the lower limb muscle groups in a sample of volleyball players could improve the explosive force of the limbs. Our working hypothesis is that osteopathic treatment, capable to reduce the stiffness of lower limbs (30), make more efficient expression of produced explosive force. Simultaneously, the osteopathic treatment would decrease spasms and tension and release tissue strain.

To this end, we have developed a protocol of manipulative techniques with the aim of improving the functionality of the lower limbs through the normalization of the fascial tissue, in order to reduce the stiffness and thus to improving the explosive force.

\section{Materials and methods}

Subjects. A total of 120 healthy semiprofessional male volleyball players were recruited into this study. We excluded athletes who had one or more of the following: scoliosis, ligamentous laxity, a history of surgery (e.g., anterior cruciate ligament reconstruction, deep scars type varicocele, appendectomy), medication use, previous ligament injuries, or a muscle-tendon with documented permanent outcomes or fractures.

Of the 120 athletes, only 57 athletes took part in the present study. The 57 athletes were a mean age $24.18( \pm 1.75)$, high $184.1 \mathrm{~cm}(1.63)$, weigh $78.12 \mathrm{~kg}( \pm 2.25)$. All 57 athletes performed three weekly training sessions and participated in a game. The subjects were fully informed of the purpose of the study and signed an informed consent document that was prepared according to the ethical standards laid down in the Declaration of Helsinki. The present research has been approved by the Ethics Committee of the Kore University of Enna. We performed a randomized controlled trial to evaluate whether fascial manipulative treatment was able to affect lower limb muscle strength. The participants were randomly divided into two groups: The fascial treatment group (FTG), which was composed of 30 athletes, and the control group (CG), which consisted of 27 athletes. Each participant was convinced to receive the same osteopathic treatment of all other subjects. In fact, the athletes of $\mathrm{CG}$ received only a non-specific surface massage.

Experimental design. The working protocol included: T0 (Fig. 1): 57 athletes participated in 10 min of warm up and stretching, before being subjected to the first battery of tests that assessed the power of the lower limbs. The sample was then divided into the FTG and CG. The FTG underwent osteopathic fascial techniques (PFT) and the CG waited in the locker room, received only a non-specific surface massage. Then, all subjects underwent a second test to measure the power of the lower limbs. T1: After seven days, the FTG was subjected to a second treatment. T2: After seven more days (i.e., after 14 days from the first treatment), the FTG was subjected to the third treatment. T3: After seven more days (i.e., after 21 days from the first treatment), the FTG was subjected to the fourth treatment. T4: After nine more days (i.e., a month later (30 days)), the first test was again conducted on both the FTG and CG groups (Fig. 1). The subjects completed a total of four treatments, with a follow-up measurement performed after 30 days of treatment.

Lower limbs power rating. The evaluation of the power of the lower limbs was conducted using jumping tests. As described by Bosco et al (31), these tests involved a platform that was placed on a rubber-coated contact mat connected to a digital timer (Ergojump, Globus Inc., Treviso, Italy). The protocol outlined in Bosco et al (31) and mathematical criteria established by Bosco et al (31-32), were followed to perform two different jumping motions: the jump squat jump (SJ) and the counter movement jump (CMJ) (33).

The increase in flight time (FT) due to the muscle pre-stretch in CMJ with respect to the jump without pre-stretch in $\mathrm{SJ}$, has repeatedly been attributed primarily to the release of elastic energy (34-36). The height of the jump was measured in centimeters. An expert in physiology and psychobiology who was blinded to the study groups measured the power of the lower limbs.

Squatting jump (SJ). This type of jump allows for the detection of the explosive force of the extensor muscles of the lower limbs. It is an easily performable and repeatable test that consists of a vertical jump at maximum intensity from the starting position, without making any downward movement (countermovement). The subject is in a starting position that includes having their feet evenly in contact with the ground. The knees are bent starting from a semisquat position with an angle of $90^{\circ}$. The opening angle of the knee in the first jump is checked with a hands goniometer. The hips and trunk are vertical to the ground during this type of jump.

Counter movement jump (CMJ). This type of jump indicates the relief of the elastic reuse capacity of the extensor muscles of the lower limbs. It consists of a vertical jump at maximum intensity, starting from the upright position, that is preceded by counter movement with bending of the knees up to approximately $90^{\circ}$ (muscle stretch-shortening cycle). The subject then falls with spread knees and lands on their toes with subsequent cushioning to prevent injury. The test was performed with the subject's hands on their hips. The subject begins in the starting position taking (as described above).

For each of the two sessions, we conducted a preliminary stage consisting of heating: i) running at a gentle pace for approximately $10 \mathrm{~min}$ on a treadmill; ii) approximately $5 \mathrm{~min}$ of stretching and iii) a test jump. After warm-up standards, all players performed on a contact-time platform (Ergojump R, Finland) following the test protocol (32).

Protocol of myofascial treatment. The myofascial treatment protocol included the execution of the below-described techniques. Each osteopathic technique was performed on 


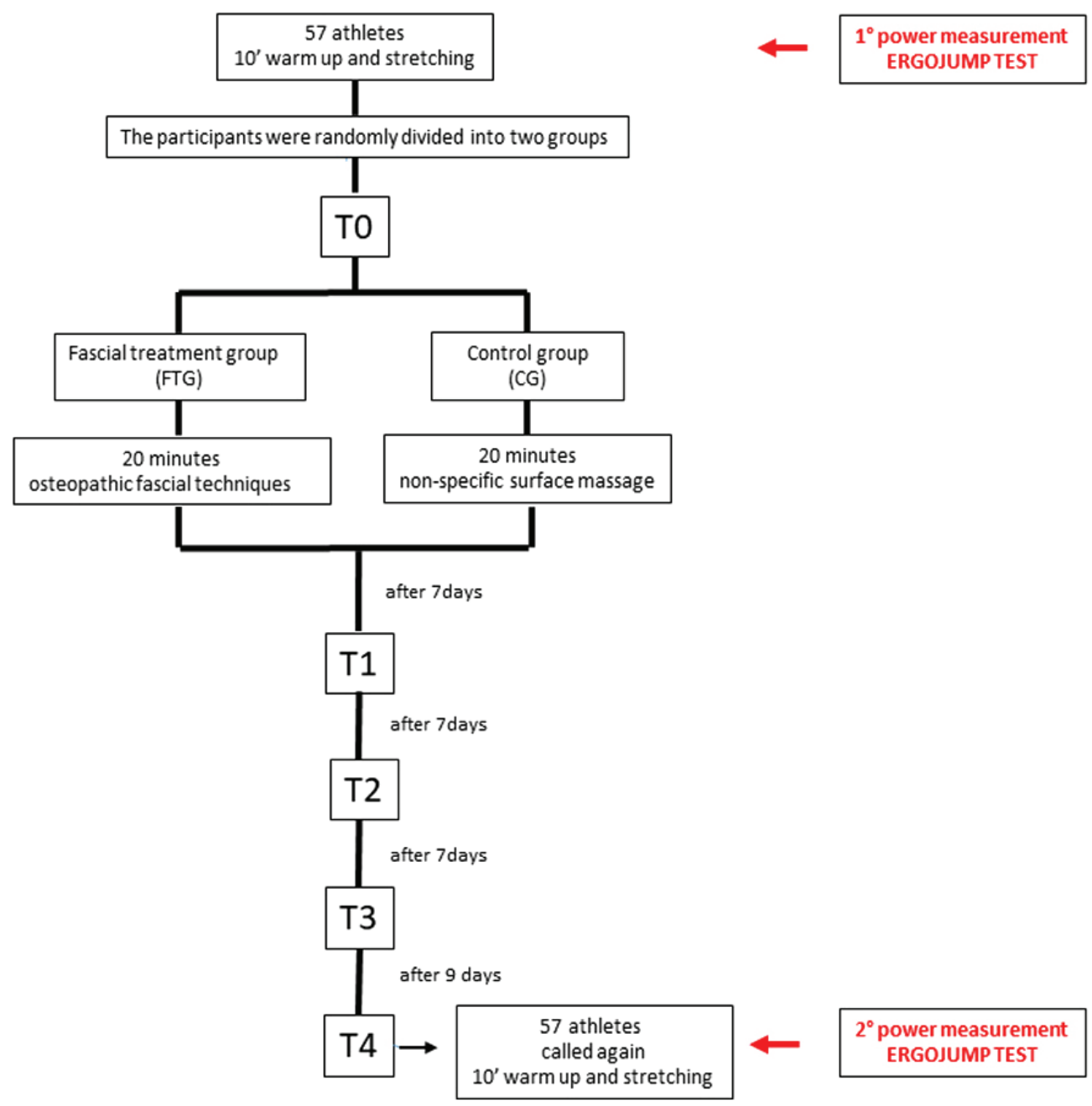

Figure 1. Experimental design.

a single point for $90 \mathrm{sec}$ and each technique performed on a single district was administered only once during the treatment session (37). The protocol was performed by a physical therapist with 14 years of experience in technical manipulation and five years of experience in osteopathic manipulation. The osteopath was not involved in measurements and was not aware of the values obtained during measurements.

Superficial dorsal fascia. The fascia that covers the tendons of the long extensor muscles, the extensor halluces longus and the anterior peroneus. The top portion of the fascia follows the annular ligament (upper and lower retinaculum mm extenders), while the inner and outer edges of fascia are along the foot and is often confused with the plantar fascia. At this point of the foot, the therapist places their fingers. With the patient in a supine position and with the osteopathic practitioner at the side of the foot, the therapist places one hand cranially to the retinacula of the ankle and the other hand is placed caudally around the metatarsal heads (Fig. 2A).

Pedidia fascia. This portion of the superficial fascia splits to cover the pedidio muscle, the pedidei vessels and nerve, and the tibialis anterior. The fascia extends to the outer edge of the foot and is often confused with the superficial fascia. The points of origin and insertion are the dorsal surface of the cuboid and the fifth toe, respectively (Fig. 2B). With the patient in a supine position, the therapist places one hand below the ankle and the other hand distal to the site of articulation with the digits. The therapist then performs a helical pulling movement.

Deep fascia. This portion of the fascia covers the metatarsal bones and the interosseous muscles. With the patient in a supine position, the osteopathic clinician sits at the feet of the patient and locks the last four digits and mobilizes the first metatarsal using up-and-down motions as well as flexed-and-extended motions. The therapist carefully tests the margin of the insertion of the metatarsal head and follows this procedure with the remaining digits (Fig. 2C).

Plantar fascia of the foot. This portion of the fascia originates from the anterior portion of the calcaneal tuberosity and extends to the metatarsal heads. With the patient in a prone position and a bent leg, the therapist sits to the side that is to be treated. The therapist then locks the heel bone and performs traction movements on the plantar fascia using a spiral motion (Fig. 2D).

Outside fascia anterior leg. This fascia follows the aponeurosis of the thigh and originates from the tuberosity of the tibia, rhyme under the joint outside of the tibia to the head 

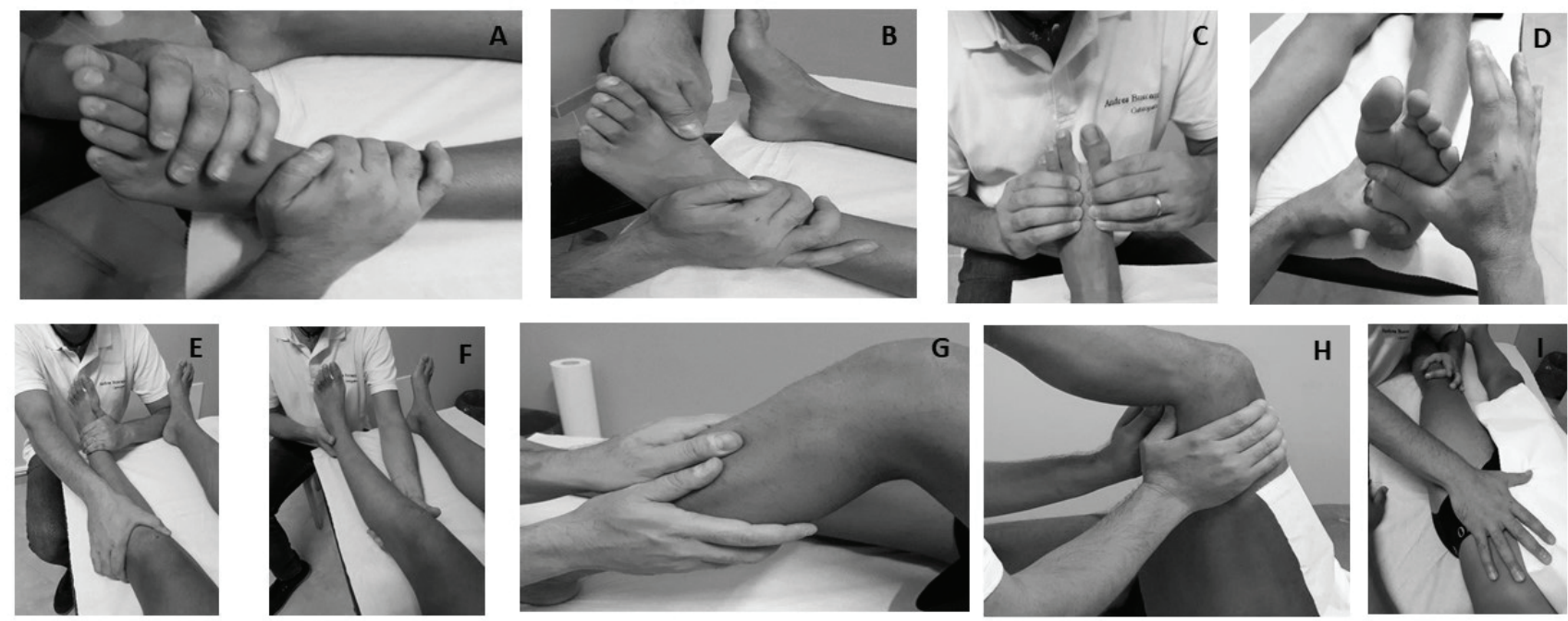

H
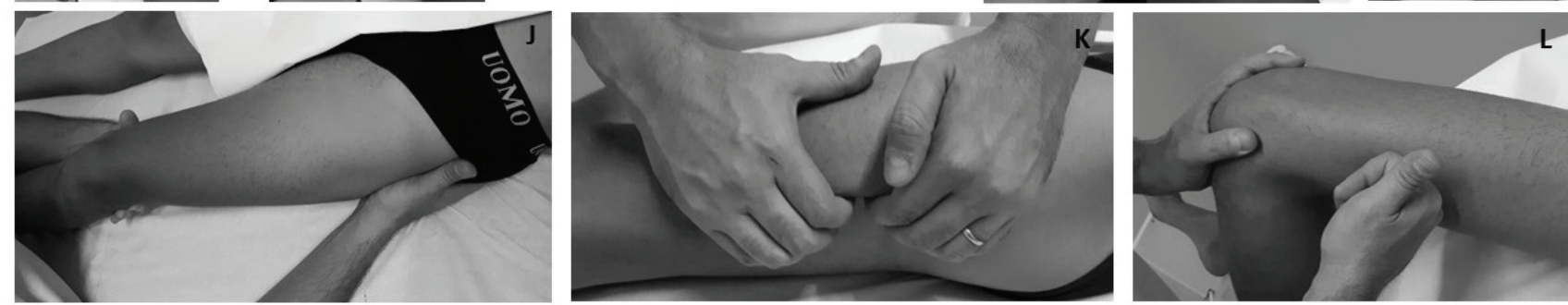

Figure 2. Protocol of myofascial treatment. (A) Superficial dorsal fascia; (B) pedidia fascia; (C) deep fascia; (D) plantar fascia of the foot; (E) outside fascia anterior leg; (F) internal fascia posterior leg; (G) interosseous membrane of the leg; (H) condyle shells; (I) front thigh superficial fascia; (J) posterior thigh superficial fascia; (K) fascia lata; and (L) fascia lata.

of the fibula in his front. At this level, it receives aponeurotic expansions of some of the thigh muscles, the biceps femoris, sartorius and semitendinosus. From its deep surface, they are detached different plates, which compose the muscle sheaths as well as intermuscular septa front and outer. With the patient in a supine position, the therapist sits with one hand below the patellar tendon and the other hand on top of the ankle. The therapist then performs helical movements (Fig. 2E).

Internal fascia posterior leg. This portion of the fascia originates at the popliteal fossa, part of the tibia, and extends to the heel pulley. With the patient in a supine position, the therapist places their hand cranially to the cable below the popliteal tuberosity of the tibia. They then place the remaining hand distally to the heel and pull without bending or stretching the foot (Fig. 2F).

Interosseous membrane of the leg. This portion of the fascia includes the tibia-peroneal interosseous membrane. It is identifiable by its course, which appears as two beams that intersect. Beams ankle peroneal: from top to bottom, from the tibia to the fibula, from the inside to inward. Beams peroneal tibia: The other at the bottom, from the fibula to the tibia, from outside to inward. This portion of the fascia also covers the tibia periosteum where the medial aspect is appreciable any may indicate possibly dysfunction. With the patient in a supine position, the therapist palpates the leg for dense areas. The therapist then runs their hand over these regions to relax them (Fig. 2G).
Condyle shells. This portion of the fascia includes a thickening of the capsule at the level of each condyle. Through its peripheral components, the condyle shells ensures the transverse stability of the knee. This is particularly evident from the inner side where the shell goes to be confused with the internal lateral ligament. With the patient in a supine position, the therapist places their hands in the popliteal area on the inter-condyle shells. The therapist then tests for tension using small thrusting motions of the hands (Fig. 2H).

Front thigh superficial fascia. This portion of the fascia includes the inguinal ligament and extends to the top margin of the patella. With the patient in a supine position, the therapist sits by the side of the limb that requires treatment. The therapist then places one hand cranially on the inguinal ligament under the anterior superior iliac spine and another hand distally above the knee. The therapist then pulls using helical movements (Fig. 2I).

Posterior thigh superficial fascia. This portion of the fascia originates from an imaginary line formed between the greater trochanter and the ischial tuberosity. It is part of the popliteal fossa above the femoral condyles. With the patient in a supine position, the therapist places one hand cranially and posteriorly to the trochanter of the femur and the other hand distally on the inter-condyle shells. The therapist then performs helical movements (Fig. 2J).

Fascia lata. With the patient in a supine position and with the therapist standing opposite the TFL that requires treatment, 

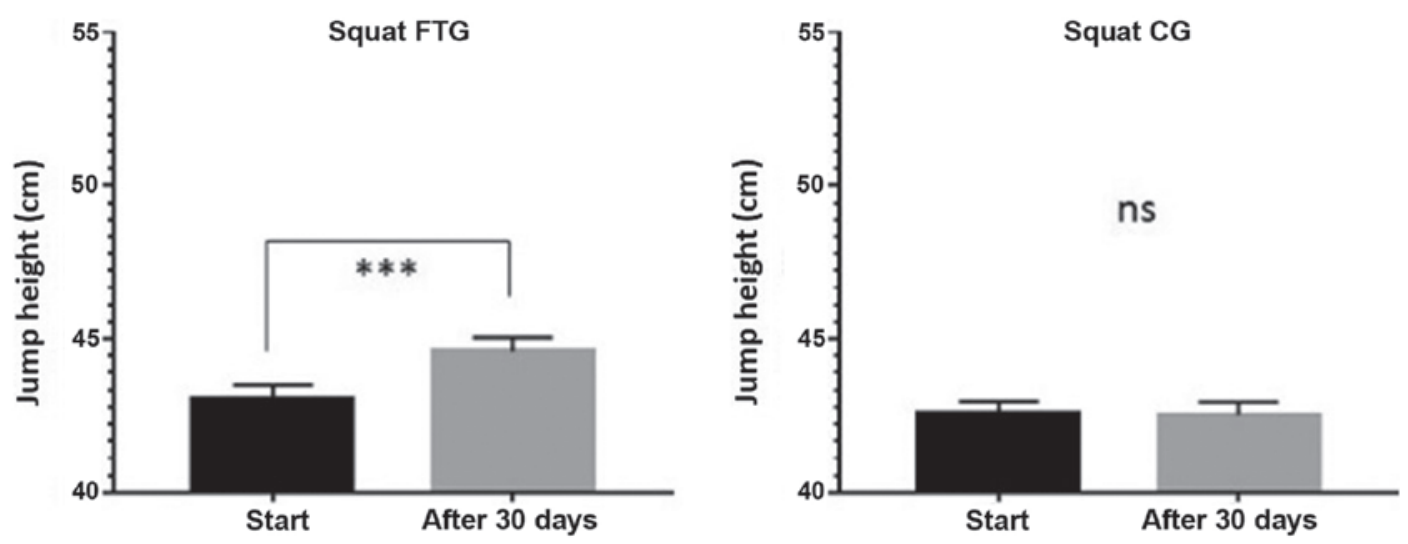

Figure 3. Results obtained during the squatting jump test $\left.{ }^{* * * *} \mathrm{P}<0.0001\right)$.
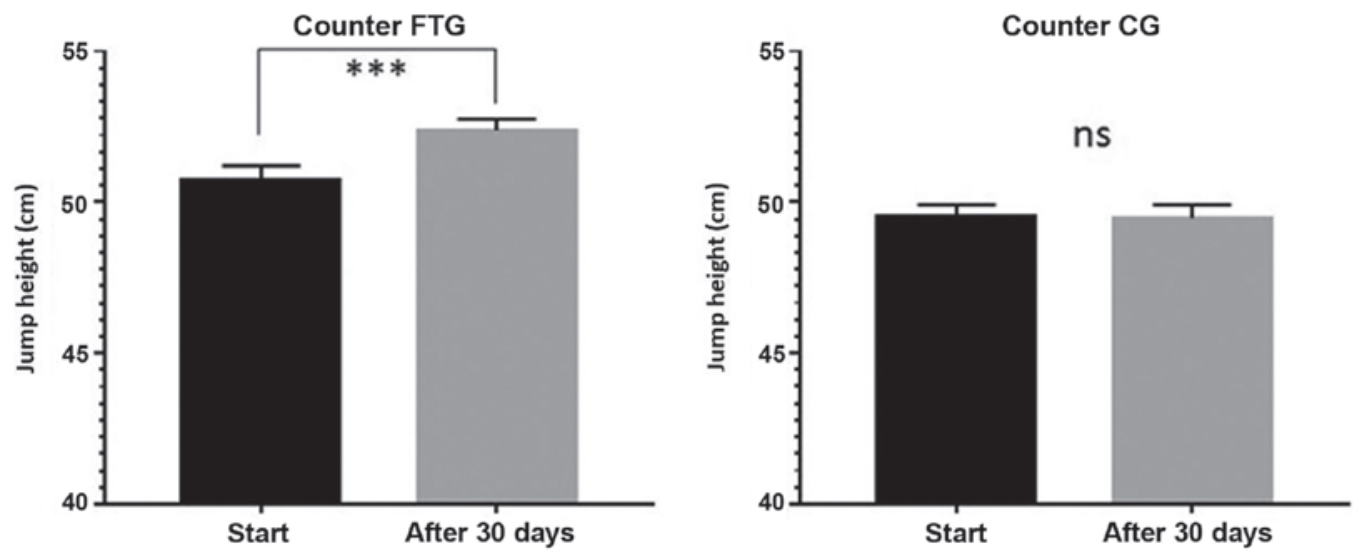

Figure 4. Results obtained in the counter movement jump test $\left.{ }^{* * * *} \mathrm{P}<0.0001\right)$.

the therapist places their hands on the muscle belly starting above the knee and then pull the muscle upward. With the patient in a supine position, the osteopathic doctor engages the fascia lata with both hands from the contralateral side and walk along its length (Fig. 2K).

Fascia lata. The portion of the fascia lata that covers the iliopsoas muscle was treated differently. With the patient in a supine position and with the therapist standing by the side of the muscle to be treated, the therapist places their hand cranially past the belly of the iliopsoas muscle, while the distal hand raises and mobilizes the ipsilateral limb, thereby creating tension in the muscle layer (Fig. 2L).

Technique 'unrolling leg fascial'. With the patient in a supine position, the therapist compresses the limb by pulling the foot while one hand is placed at the knee to aid in mobilization of the entire limb.

Statistical analysis. We performed an ANOVA (Friedman test), which was followed by Dunn's multiple comparison test. All data are reported as the mean \pm standard deviation and $\mathrm{P}<0.05$ was considered to indicate a statistically significant difference. Analyses were performed using GraphPad Prism v.6.0 for Windows (GraphPad Software, Inc., La Jolla, CA, USA).

\section{Results}

The baseline measurements indicated that the sample was homogeneous relative to strength with the athletes obtaining average values of 42.49 and $48.54 \mathrm{~cm}$ in the SJ test and in the CMJ, respectively.

The results obtained during the SJ test are shown in Fig. 3. The FTG exhibited a statistically significant improvement in the values obtained before and after 30 days, with the measurements increasing from $43.08 \pm 0.42 \mathrm{~cm}$ at baseline to $44.6 \pm 0.44 \mathrm{~cm}$ after 30 days $(\mathrm{P}<0.0001)$. Conversely, there was no improvement in the $\mathrm{CG}$, with the measurements increasing from $42.56 \pm 0.40 \mathrm{~cm}$ at baseline to $42.52 \pm 0.41$ after 30 days $(\mathrm{P}<0.56)$.

The results obtained in the CMJ test are shown in Fig. 4. The FTG exhibited a statistically significant improvement in the values obtained before and after 30 days, with the measurements increasing from $50.72 \pm 0.49 \mathrm{~cm}$ at baseline to $52.38 \pm 0.37 \mathrm{~cm}$ after 30 days $(\mathrm{P}<0.0001)$. Meanwhile, there was no improvement in the $\mathrm{CG}$, with the measurements increasing from $49.48 \pm 0.43 \mathrm{~cm}$ at baseline to $49.48 \pm 0.44 \mathrm{~cm}$ after 30 days $(\mathrm{P}<0.32)$.

\section{Discussion}

The findings from this research show that that removing the somatic dysfunction borne by the fascial system of the lower 
limbs resulted in an improvement in performance by increasing the explosive force. These results were in line with the study by Brown et al (38) as well as Tozzi et al (39), who hypothesized that connective tissue has a specific role in both the generation and transfer of force (40).

From a physiological point of view it could be suggested the observed improvement in performance could be due, at least in part, to a significant changes of stiffness of lower limbs. Stiffness can be defined as 'the property of a system to resist an applied stretch' and can be calculated by dividing the variation in force by the variation in length $(\mathrm{DF} / \mathrm{DL})$ of the system of interest (41). The concept of leg-spring stiffness describes an integration of the stiffness of all structures (muscles, tendons, and ligaments acting across joints) of lower limb during movement and defines the capability of those structures to act in unison in a springlike manner (42). The improvements that we observed indicated that myo-fascial treatment stimulates the fascia, thereby facilitating the reaction of connection tissue and resulting in improvements.

Thirty days of treatment led us to hypothesize that the metabolic activity of the fascia can, over time, restore the body to optimal biomechanical conditions. We further determined that correction of the fascial system requires a relatively long period of time to obtain the best results.

The observed improvement of $2 \mathrm{~cm}$ in the measurement of jump in athletes treated with osteopathic techniques has a significant clinical relevance as it shows that, with equal training, the treated group has a better response to the tone/trophism of lower limbs' musculature.

Osteopathic tests and manipulative techniques facilitated the troubleshooting and resolution of somatic dysfunction. It also improved the mechanical function of the organism. Muscles contract actively to generate force, which they then transfer by deforming the surrounding connective tissue. This action influences the application of force by modifying the magnitude and direction of connective tissue deformation (43-46) and, therefore, of stiffness. The introduction of a fascial protocol during athletic training can improve the balance of the bands and, as a direct result, improve the performance and efficiency of the musculoskeletal system, thereby reducing the risk of injury. The strong point of the job is certainly to have put in place a protocol of techniques that allow to investigate in a sectoral and specific way the whole fascial system of the lower limb. A weak point of the work could be the athlete, if you did not perform the jump properly. In this way, would to may be to perform osteopathic techniques every two months during an athletic season so as to maintain the balance of the fascial system and obtain excellent performance.

The purpose of this study was largely achieved the sample of volleyball players that has been subjected to osteopathic treatment of the lower limb muscle groups has shown an improvement the explosive force of the limbs, while reducing spasms and tension, releasing tissue strain, and correcting posture. The strength of the present work is undoubtedly the development of a detailed and widely reproducible protocol both in term of techniques and timing. The applicability of this protocol is twofold: in a clinical field, to recover post-traumatic tissue damages with residual sore, and in a sports setting to improve athlete's performances. Future research is required to develop a new treatment protocol suitable for the upper limbs and trunk.

\section{Acknowledgements}

The study was supported and conducted by a multi-disciplinary team with the collaboration of CSDOI (Centro Studi Osteopatia Italiano) of Catania, Italy.

\section{Funding}

No funding was received.

\section{Availability of data and materials}

The datasets used and/or analyzed during the current study are available from the corresponding author on reasonable request.

\section{Authors' contributions}

$\mathrm{AB}, \mathrm{CP}$ and $\mathrm{CM}$ conceived and designed the experiments. $\mathrm{AB}$, $\mathrm{CP}$ and $\mathrm{CM}$ performed the experiments. TR, AB, MCP, AR, $\mathrm{CP}, \mathrm{ViP}, \mathrm{VaP}, \mathrm{DDC}$ and $\mathrm{CM}$ analyzed the data. AB and CM wrote the paper.

\section{Ethics approval and consent to participate}

The paper was approved by the Internal Review Board of the Research in Psychology at the Kore University of Enna (Enna, Italy). The subjects signed an informed consent document that was prepared according to the ethical standards laid down in the Declaration of Helsinki.

\section{Consent for publication}

Written informed consent was provided by all subjects.

\section{Competing interests}

The authors declare that they have no competing interests.

\section{References}

1. Bordoni B and Zanier E: Skin, fascias, and scars: Symptoms and systemic connections. J Multidiscip Healthc 7: 11-24, 2013.

2. Bordoni B and Zanier E: Cranial nerves XIII and XIV: Nerves in the shadows. J Multidiscip Healthc 6: 87-91, 2013.

3. Abbott RD, Koptiuch C, Iatridis JC, Howe AK, Badger GJ and Langevin HM: Stress and matrix-responsive cytoskeletal remodeling in fibroblasts. J Cell Physiol 228: 50-57, 2013.

4. van der Wal J: The architecture of the connective tissue in the musculoskeletal system-an often overlooked functional parameter as to proprioception in the locomotor apparatus. Int J Ther Massage Bodywork 2: 9-23, 2009.

5. Stecco A, Gesi M, Stecco C and Stern R: Fascial components of the myofascial pain syndrome. Curr Pain Headache Rep 17: 352, 2013

6. Buckley CD: Why does chronic inflammation persist: An unexpected role for fibroblasts. Immunol Lett 138: 12-14, 2011.

7. Tozzi P: Selected fascial aspects of osteopathic practice. J Bodyw Mov Ther 16: 503-519, 2012.

8. Branislav R, Milivoj D, Abella CP, Deval VC and Siniša K: Effects of combined and classic training on different isometric rate of force development parameters of leg extensors in female volleyball players: Discriminative analysis approach. J Res Med Sci 18: 840-847, 2013. 
9. Guimberteau JC, Delage JP, McGrouther DA and Wong JK: The microvacuolar system: How connective tissue sliding works. J Hand Surg Eur Vol 35: 614-622, 2010.

10. Turrina A, Martínez-González MA and Stecco C: The muscular force transmission system: Role of the intramuscular connective tissue. J Bodyw Mov Ther 17: 95-102, 2013.

11. Purslow PP: Muscle fascia and force transmission. J Bodyw Mov Ther 14: 411-417, 2010.

12. Tozzi P: A unifying neuro-fasciagenic model of somatic dysfunction-underlying mechanisms and treatment-part I. J Bodyw Mov Ther 19: 310-326, 2015.

13. Tozzi P: A unifying neuro-fasciagenic model of somatic dysfunction-underlying mechanisms and treatment-part II. J Bodyw Mov Ther 19: 526-543, 2015.

14. Giacomelli IL, Steidle LJ, Moreira FF, Meyer IV, Souza RG and Pincelli MP: Hospitalized patients with COPD: Analysis of prior treatment. J Bras Pneumol 40: 229-237, 2014 (In English, Portuguese).

15. Stendardi L, Grazzini M, Gigliotti F, Lotti P and Scano G: Dyspnea and leg effort during exercise. Respir Med 99: 933-942, 2005.

16. Savage PA, Shaw AO, Miller MS, VanBuren P, LeWinter MM, Ades PA and Toth MJ: Effect of resistance training on physical disability in chronic heart failure. Med Sci Sports Exerc 43: 1379-1386, 2011

17. Erbs S, Höllriegel R, Linke A, Beck EB, Adams V, Gielen S, Möbius-Winkler S, Sandri M, Kränkel N, Hambrecht R and Schuler G: Exercise training in patients with advanced chronic heart failure (NYHA IIIb) promotes restoration of peripheral vasomotor function, induction of endogenous regeneration, and improvement of left ventricular function. Circ Heart Fail 3: 486-494, 2010

18. Mastaglia FL: The relationship between muscle pain and fatigue. Neuromuscul Disord 22 (Suppl 3): S178-S180, 2012.

19. Voelzke M, Stutzig N, Thorhauer HA and Granacher U: Promoting lower extremity strength in elite volleyball players: Effects of two combined training methods. J Sci Med Sport 15: 457-462, 2012

20. Forthomme B, Croisier JL, Ciccarone G, Crielaard JM and Cloes M: Factors correlated with volleyball spike velocity. Am J Sport Med 33: 1513-1519, 2005.

21. Rodriguez-Ruiz D, Quiroga ME, Miralles JA, Sarmiento S, de Saá Y and García-Manso JM: Study of the technical and tactical variables determining set win or loss in top-level European men's volleyball. J Quant Anal Sport 7: 7-15, 2011.

22. Marcelino R, Mesquita I and Afonso J: The weight of terminal actions in volleyball. Contributions of the spike, serve and block for the team's rankings in the World League 2005. Int J Perf Anal Sport 8: 1-7, 2008.

23. Fry A, Kraemer WJ, Waseman CA, Conroy BP, Gordon SE, Hoffman JR and Maresh CM: The effects of an off-season strength and conditioning program on starters and non-starters in women's intercollegiate volleyball. J Strength Cond Res 5: 174-181, 1991.

24. Sheppard JM, Cronin J, Gabbett TJ, McGuigan MR, Etxebarria N and Newton RU: Relative importance of strength, power, and anthropometric measures to jump performance of elite volleyball players. J Strength Cond Res 22: 758-765, 2008.

25. Sheppard JM, Gabbett T, Taylor KL, Dorman J, Lebedew AJ and Borgeaud R: Development of a repeated-effort test for elite men's volleyball. Int J Sports Physiol Perform 2: 292-304, 2007.

26. Sheppard JM and Borgeaud R: Influence of stature on movement speed and repeated efforts in elite volleyball players. J Aust Strength Cond 16: 12-24, 2008.

27. Sheppard JM, Dingley AA, Janssen I, Spratford W, Chapman DW and Newton RU: The effect of assisted jumping on vertical jump height in high-performance volleyball players. J Sci Med Sport 14: 85-89, 2011.

28. Newton RU, Kraemer WJ and Häkkinen K: Effects of ballistic training on preseason preparation of elite volleyball players. Med Sci Sports Exerc 31: 323-330, 1999.
29. Newton RU, Rogers RA, Volek JS, Häkkinen K and Kraemer WJ: Four weeks of optimal load ballistic resistance training at the end of season attenuates declining jump performance of women volleyball players. J Strength Cond Res 20: 955-961, 2006.

30. Xu Q, Chen B, Wang Y, Wang X, Han D, Ding D, Zheng Y, Cao Y, Zhan $\mathrm{H}$ and Zhou Y: The effectiveness of manual therapy for relieving pain, stiffness and dysfunction in knee osteoarthritis: A systematic review and meta-analysis. Pain Physician 20: 229-243, 2017.

31. Bosco C, Luhtanen P and Komi PV: A simple method for measurement of mechanical power in jumping. Eur J Appl Physiol Occup Physiol 50: 273-282, 1983.

32. Komi PV and Bosco C: Utilization of stored elastic energy in leg extensor muscles by men and women. Med Sci Sports 10: 261-265, 1978.

33. Baker D: Improving vertical jump performance through general, special and specific strength training: A brief review. J Strength Cond Res 10: 131-136, 1996.

34. Häkkinen K: Neuromuscular and hormonal adaptations during strength and power training. A review. J Sports Med Phys Fitness 29: 9-26, 1989.

35. Lidor R, Hershko Y, Bilkevitz A, Arnon M and Falk B: Measurement of talent in volleyball: 15-month follow-up of elite adolescent players. J Sports Med Phys Fitness 47: 159-168, 2007.

36. Marina M, Jemni M and Rodríguez F: Jumping performance profile of male and female gymnasts. J Sports Med Phys Fitness 53: 378-386, 2013.

37. Zein-Hammoud M and Standley PR: Modeled osteopathic manipulative treatments: A review of their in vitro effects on fibroblast tissue preparations. J Am Osteopath Assoc 115: 490-502, 2015.

38. Brown SH, Carr JA, Ward SR and Lieber RL: Passive mechanical properties of rat abdominal wall muscles suggest an important role of the extracellular connective tissue matrix. J Orthop Res 30: 1321-1326, 2012.

39. Tozzi P, Bongiorno D and Vitturini C: Low back pain and kidney mobility: Local osteopathic fascial manipulation decreases pain perception and improves renal mobility. J Bodyw Mov Ther 16: 381-391, 2012.

40. Ćosić V, Day JA, Iogna P and Stecco A: Fascial manipulation ${ }^{(\circledR)}$ method applied to pubescent postural hyperkyphosis: A pilot study. J Bodyw Mov Ther 18: 608-615, 2014.

41. Saez Saez de Villarreal E, González-Badillo JJ and Izquierdo M: Optimal warm-up stimuli of muscle activation to enhance short and long-term acute jumping performance. Eur J Appl Physiol 100: 393-401, 2007.

42. Grange RW, Cory CR, Vandenboom R and Houston ME: Myosin phosphorylation augments force-displacement and force-velocity relationships of mouse fast muscle. Am J Physiol 269: C713-C724, 1995.

43. Allen SH: Primary osteoporosis. Methods to combat bone loss that accompanies aging. Postgrad Med 93: 43-46, 49-50, 53-55, 1993.

44. Willard FH, Vleeming A, Schuenke MD, Danneels L and Schleip R: The thoracolumbar fascia: Anatomy, function and clinical considerations. J Anat 221: 507-536, 2012.

45. Lunghi C, Tozzi P and Fusco G: The biomechanical model in manual therapy: Is there an ongoing crisis or just the need to revise the underlying concept and application? J Bodyw Mov Ther 20: 784-799, 2016.

46. Klingler W, Velders M, Hoppe K, Pedro M and Schleip R: Clinical relevance of fascial tissue and dysfunctions. Curr Pain Headache Rep 18: 439, 2014.

This work is licensed under a Creative Commons Attribution-NonCommercial-NoDerivatives 4.0 International (CC BY-NC-ND 4.0) License. 